\title{
Effect of Parallel Talk on the Language and Interactional Skills of Preschoolers with Cochlear Implants and Hearing Aids
}

\author{
Sharon A. Raver \\ Old Dominion University \\ Norfolk, VA USA \\ Peggy Hester \\ Old Dominion University
}

\author{
Jonna Bobzien \\ Old Dominion University
}

Anne Michalek

Old Dominion University

\author{
Corrin Richels \\ Old Dominion University
}

Nicole Anthony
Old Dominion University

\begin{abstract}
Children with profound congenital hearing loss often do not have the same prelinguistic opportunities for social and verbal interaction as their peers with typical hearing [14]. Consequently, language and social skills may be challenging for this group, even after they are provided with amplification or a cochlear implant. This pilot study examined the effectiveness of using a parallel talk intervention to increase the language and interactional skills of three preschoolers with deafnesss. Results revealed that all participants increased verbal turn-taking and that two of the three increased initiated and responded vocal/verbal comments, and initiated and responded nonverbal responses during a 5-minute play session in which parallel talk was utilized. Additionally, all children displayed some generalization in the two types of generalization probes employed. Implications for facilitating the communication of preschoolers with communication and social delays are discussed.
\end{abstract}

\section{Introduction}

According to Geers and colleagues [5], children with profound hearing loss who do not receive cochlear implants (CI) could be expected to acquire language at about half the rate of their same-aged peers. Nonetheless, CIs do not create sound perception that is the same as normal hearing. Therefore, individuals who use CIs, and/or hearing aids, require intensive habilitation programs [2]. The social skills of children who are deaf are particularly impacted even when they receive CIs and/or amplification [14]. This finding highlights the need for intervention that targets language skills in the context of social interaction.

\section{Literature Review}

\subsection{Children with CI and Social Interaction}

Although many children with CIs are able to eventually learn language, the CI cannot "make-up" for language learning opportunities missed by the child prior to implantation. Research [12] supports the idea that prelinguistic skills, and especially the development and impact of joint attention, are dependent on factors within the child and within the child's immediate environment. These children tend to lag behind their age-appropriate peers in their ability to use appropriate pragmatic skills. Most notably, turn-taking in the form of reciprocity of joint attention with caregivers is problematic when so much of these early interactions require being able to localize and respond to auditory information [17]. An infant with a profound hearing loss does not have access to auditory cues in his or her environment including a caregiver's speech. This is especially critical since a child's ability to respond to caregiver bids for joint attention is a predictor of receptive language at 18- to 21-months [12] and 29-months [10]. Consequently, responding to joint attention is a prelinguistic skill that hearing children develop before the age that most children would receive their CIs [16]. As a result, even after receiving a CI, it is possible that the pragmatic abilities of these children continue to be impacted by the lack of initial experiences with basic verbal turn-taking patterns and joint attention. There is promising research, however, which shows that following implantation, maternal sensitivity to opportunities for communication with their toddlers improves [1]. Thus, it is important to continue to target these prelinguistic skills following implantation and after verbal language has begun to develop. 
For young children, play is the primary mode of social exchange [7]. During play, parents, caregivers or teachers, have the opportunity to model vocabulary, grammar, and social interaction strategies for children. There are many naturalistic social-communicative techniques used to scaffold verbal skills during play, including recast therapy [3], enhanced milieu teaching [9], as well as parallel and self-talk [6], [7]. Teachers and speech-language pathologists report these types of child-directed interactions facilitate language skills in preschoolaged children who are typically developing as well as those who are not [11]. Despite the fact that these strategies are commonly used together to create interventions for children with delayed communication skills, a literature search yielded no empirical evidence that they support children's language skills when used independently.

Parallel talk is an intervention strategy in which an interactional partner comments on a child's play by stating what the child is doing, thinking or feeling, rather than requiring the child to answer direct questions or produce particular responses [7]. Additionally, no particular communication targets are specified. This strategy is child-directed in that it does not require the child to make a response or repeat a particular verbal model. Parallel talk creates joint attention between the adult and child, a basic component of conversations with young children. Because children with hearing loss may display persistent difficulties with social and verbal skills, parallel talk seems to offer promise as a technique that may facilitate natural opportunities for conversational turn-taking and joint attention. Although there is some clinical support for the value of this strategy, empirical support is lacking.

\section{Research Rationale}

The purpose of this pilot study was to determine if an adult's use of parallel talk during play increased the verbal turn-taking, verbal comments, and imitative responses of preschool-aged children with hearing loss and cochlear implants and/or hearing aids. Further, the study examined if generalization of these skills occurred immediately after parallel talk was used and if targeted behaviors transferred to an unstructured play session with another child when an adult was not present.

\section{Method}

Three preschool children, two boys and one girl, with deafness who had received cochlear implants or amplification through hearing aids, participated. Participants were chosen from an oral-language public school classroom in which only speaking and listening approaches were used. The class served six preschoolers with hearing loss. Participants met the following inclusion criteria: (1) a minimum of one year delay in expressive and receptive language skills, (2) at least one year delay in pragmatic skills involving initiating, maintaining, and concluding a conversation appropriately with adults/peers, (3) had cochlear implant(s) and/or hearing aid(s), and (4) each parent gave written consent for participation.

Table 1. A Summary of Participants’ Characteristics

\begin{tabular}{|l|l|l|l|}
\hline Child & Sex & $\begin{array}{l}\text { Auditory } \\
\text { Supports }\end{array}$ & $\begin{array}{l}\text { Speech \& Language } \\
\text { Characteristics }\end{array}$ \\
\hline 1 & M & $\begin{array}{l}\text { Hearing } \\
\text { aid left } \\
\text { ear; } \\
\text { cochlear } \\
\text { implant } \\
\text { on right } \\
\text { ear }\end{array}$ & $\begin{array}{l}\text { Receptive/Expressive } \\
\text { language delays; } \\
\text { MLU 3.0; 45\% } \\
\text { intelligibility with a } \\
\text { familiar speaker. }\end{array}$ \\
\hline 2 & F & $\begin{array}{l}\text { Bilateral } \\
\text { hearing } \\
\text { aids }\end{array}$ & $\begin{array}{l}\text { Receptive/Expressive } \\
\text { language delays; } \\
\text { MLU 1.95; 75\% } \\
\text { intelligibility with a } \\
\text { familiar speaker. }\end{array}$ \\
\hline 3 & M & $\begin{array}{l}\text { Cochlear } \\
\text { implant } \\
\text { on right } \\
\text { ear }\end{array}$ & $\begin{array}{l}\text { Receptive/Expressive } \\
\text { language delays; } \\
\text { MLU 2.16, 50\% } \\
\text { intelligibility with a } \\
\text { familiar speaker. }\end{array}$ \\
\hline
\end{tabular}

Child 1 was a 4-year 5 month old Caucasian male diagnosed at 1-year 6 months with a moderate to severe bilateral hearing loss who began wearing bilateral hearing aids at the time of diagnosis. He received a right ear cochlear implant when he was 3 years old. On the Preschool Language Scale-4 (PLS4 ) he scored a total language age equivalent of 2-8 (auditory comprehension age equivalent 3-1; expressive communication age equivalent 2-7).

Child 2 was a 3 -year 7 month old Caucasian female diagnosed at 3 months with severe bilateral sensorineural hearing loss. She received analog hearing aids at the age of 12 months but did not consistently wear them until she was about 2-years 6 months old. According to the Preschool Language Scale-4 (PLS-4), she had a total language age 
equivalent of 1-11 (auditory comprehension age equivalent 1-11; expressive communication age equivalent 1-11).

Child 3 was a 5-year 3 month old Caucasian male diagnosed at birth with bilateral profound hearing impairment, visual impairment (Coloboma Micro-Opthamalmia), and hypotonia. He began wearing hearing aids at 10 months of age and received a cochlear implant in his right ear when he was 2-years, 10 months old. According to results on the Preschool Language Scale-4 (PLS-4), he had a total language age equivalent of 2-3 (auditory comprehension age equivalent 2-3; expressive communication age equivalent 2-3).

The classroom teacher was a Caucasian female who held a master's degree in Education of the Deaf, and held a Listening and Spoken Language (LSLC AVEd) certification from AG Bell. Despite the fact that the teacher and the two paraeducators in the classroom agreed to follow the research team's suggestions, they were blind to the study's research questions.

\subsection{Experimental Design}

An single-subject multiple baseline design across participants design, with two types of generalization probes embedded, was used. To demonstrate experimental control, a baseline was established for each participant concurrently, and once a stable trend was observed, the independent variable, the use of parallel talk, was introduced to each participant sequentially, beginning with Child 1 and concluding with Child 3. The study was conducted for 26-29 sessions over 15 weeks.

Data were collected on videotapes made by a Flip Camera placed unobtrusively on a small tripod in the classroom. The frequency of five dependent variables of communicative and pragmatic behaviors were measured and defined as the following: 1) vocal/verbal turn-taking (a comment or verbal approximation made in response to an adult's comment or a comment or verbal approximation that indicated by the child's face or voice that he/she was expecting a response), 2) initiated and responded verbal/vocal comments (an initiation or response by the child that resulted in any of the following ontopic comments: a) vocal/verbal approximation, b) a comment/statement, or c) a command/request for adult, peer, or self to do something), 3) initiated and responded nonverbal responses (a gestural or physical response that indicated that a child understood an adult's or peer's comment, or a nonverbal request for an object or an action), 4) imitative utterances of adult (an imitated utterance that included any part of what the adult had said by using the adult's word(s), including prosody, intonation and pitch, within 5 seconds of an adult's utterance), and 5) vocal/verbal questions (an utterance in which a child requested information, an object, or an action from the adult, using rising intonation or a gesture). A child had to make visual and/or physical contact with an adult or peer to have any of these behaviors coded.

The independent variable was the use of the language facilitation strategy called parallel talk. Parallel talk was defined as the process of an adult describing a child's actions, emotions, activities, and gestures, using language that linguistically matched the child's communication level [6], [7]. During intervention play sessions, an adult verbally commented on a child's activities, matched the child's nonverbal communicative intentions with verbal language, used syntactical input that closely matched a child's syntactical level, and described how the child was feeling (e.g., happy, sad, frustrated) [4].

\subsection{Procedures}

The study was conducted in an oral preschool program which met 5 days a week and had activity centers, a kitchen area, a snack/work area, and a group area. In this program an auditory-verbal approach to communication was taught. In the baseline phase, one of the paraeducators accompanied a child to a table away from the other children, and turned on the camera. The participant was then told to play with the preselected materials until the timer rang (5 minutes). Materials were table toys such as interconnecting construction materials, cars and a garage, leggos, and a doctor's kit. The paraeducator sat next to the participant, slightly to the front and right of the child, to foster eye contact. Help was given if a participant indicated a need, but no verbal models, questions, and comments were provided. The paraeducator silently watched the participant's play. Materials were changed each session. The same procedures were followed for each participant.

Before the intervention phase, the paraeducators received three 45-minute training sessions. During training, the paraeducators took turns role-playing the participant role so each had experience spontaneously offering statements that were syntactically matched to each participant's communication abilities. Training continued until each paraeducator was able to use parallel talk as defined by the protocol, and use the appropriate syntactical level for each participant for two consecutive sessions, with $100 \%$ accuracy.

For intervention, the paraeducators followed the same procedures used in baseline, and also 
introduced the use of parallel talk by describing the child's actions, emotions, activities and gestures, at the child's syntactical level, pausing at least 10 seconds between statements. The paraeducator did not ask questions, but answered questions if they occurred. No praise was offered. Generally, sessions were held 3 times a week, although this varied slightly due to scheduling conflicts. The paraeducators provided 19-30 comments during each session.

Generalization 1 monitored the effects of the intervention immediately following training. A paraeducator reset to the timer for 4 minutes after it rang and said the following: "You can play some more with __. I am still going to sit with you." The paraeducator did not use parallel talk or provide any communication support, although she answered questions and gave information if it was requested, and then returned to watching the participant play. This type of probe occurred approximately once every two weeks.

Generalization 2 monitored intervention effects across situations, materials and individuals. In this probe, two children, a participant and another child, were told that they were to play together at the table with a selected material until the timer rang (5 minutes). The paraeducators helped other children in the classroom and did not provide any communication support or praise. This probe used the same materials that had been used during intervention that day. These probes occurred approximately once every two weeks.

Treatment fidelity data was completed on $37 \%$ of the recorded sessions. Observations were designed to determine adherence to the implementation steps designated on the treatment protocol checklist for each condition. This was derived by dividing the total number of steps in the protocol by the number of steps that did not follow the protocol, and multiplying by a 100 . The mean for implementation fidelity was $100 \%$.

\subsection{Interrater Reliability}

Interrater observer agreement percentages were calculated on $34 \%$ of the videotaped sessions for Child 1, 30\% of the sessions for Child 2, and 29\% of the sessions for Child 3. Interrater agreement was determined by dividing the total number of agreements by the number of disagreements plus agreements, and multiplying by 100 . Overall, the mean interrater reliability for Child 1 was $97 \%$ (range $=90-100 \%$ ), Child 2 was 98\% (range $=93-$ $100 \%$ ), and Child 3 was $97 \%$ (range $=86-100 \%$ ) for all conditions and target behaviors.

\section{Results}

Results are presented in three sections: intervention, generalization 1, and generalization 2 .

\subsection{Intervention}

As a consequence of intervention, all three participants experienced an increase in vocal/verbal turn-taking during table play. Additionally, an increased rate of initiated and responded verbal/vocal comments and initiated and responded nonverbal responses were produced by two of the three participants when compared to baseline levels, while a low rate of imitative utterances and vocal/verbal questions were produced by all.

The most frequently occurring communication and interactional skill was vocal/verbal turn-taking. Child 1 produced a mean of 1 occurrence of vocal/verbal turn-taking (range $=0-1$ ) during 4 baseline sessions and an increased mean of 10.7 (range $=4-21$ ) during 26 intervention sessions. Child 2 produced a mean of .17 occurrences of vocal/verbal turn-taking (range $=0-1$ ) during 6 baseline sessions and an increased mean of 5.68 (range $=1-10$ ) during 20 intervention sessions. Child 3 produced a mean of 5.14 occurrences of vocal/verbal turn-taking (range=1-11) during 7 baseline sessions and an increased mean of 11.25 (range=2-26) during 20 intervention sessions.

Both Child 1 and Child 2 demonstrated an increase in the frequency of initiated and responded verbal/vocal comments, from baseline to intervention. Child 1 produced a mean of 6.00 $\mathrm{vocal} /$ verbal comments (range $=4-8$ ) during baseline and an increased mean of 8.91 (range $=2-19$ ) during intervention. Child 2 produced a mean of .33 vocal/verbal comments (range $=0-2$ ) during baseline and an increased mean of 2.68 (range $=0-7$ ) during intervention. Child 3 did not display an increase in total initiated and responded vocal/verbal comments during baseline (mean 11.71; range $=6-21$ ) or intervention (mean 9.75; range=2-28). Despite this, Child 3 did show a slight increase in responded vocal/verbal comments when baseline and intervention levels were compared (baseline mean 1.85; range $=0-4$ and intervention mean 2.947; range $=0-7$ ).

Similar to the vocal/verbal comments results, Child 1 and Child 2 demonstrated an increase in nonverbal initiations and responses when compared to their baseline levels. Child 1 produced a mean of .25 of total nonverbal initiations and responses (range $=0-1$ ) during baseline and a mean of 1.22 (range $=0-6$ ) during intervention. Child 2 produced 
mean of .14 nonverbal initiations/responses (range $=0-1$ ) during baseline and a mean of 2.47 (range $=0-8$ ) during intervention. Child 3 did not display an increase in nonverbal initiations and responses (baseline mean 2.29; range=1-6) and intervention mean of 1.17 (range $=0-3$ ).

A low rate of imitative utterances and vocal/verbal questioning was demonstrated by all participants. The baseline range for imitative utterances for the 3 participants was $0-1$ and $0-2$ in intervention. The baseline range for vocal/verbal questions was 0 for all participants and $0-1$ in intervention. Figure 1 shows the participants' vocal/verbal turn-taking.

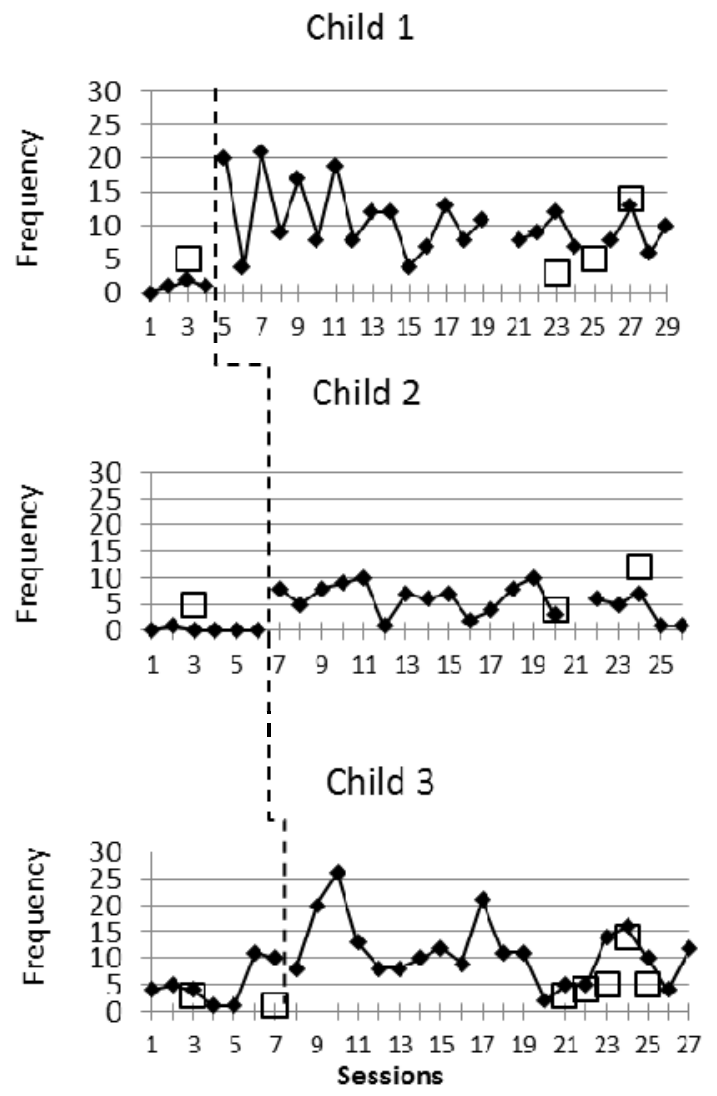

Generalization 2 Probes, 5-minute play session with another child during a different time of the school day.

Figure 1. Frequency of Vocal/Verbal Turn-Taking During Baseline and Intervention, by Participant

\subsection{Generalization 1}

The effects of the use of parallel talk immediately following intervention were measured by Generalization 1 probes. All three participants maintained their increased levels of vocal/verbal turn-taking when compared to their individual baseline means during these probes. During the four Generalization 1 probes, a mean of $6.0 \mathrm{vocal} /$ verbal turn-taking occurrences were produced by Child 1 (range=3-9). A mean of $2.0 \mathrm{vocal} /$ verbal turn-taking occurrences were produced by Child 2 (range $=1-3$ ). During three Generalization 1 probes, a mean of 11.6 occurrences of vocal/verbal turn-taking were produced by Child 3 (range $=5-23$ ).

Additionally, both Child 1 and Child 2 maintained increased levels of total initiated and responded vocal/verbal comments and nonverbal initiations and responses. Child 1 produced a mean of 9.8 $\mathrm{vocal} / \mathrm{verbal}$ comments (range $=0-11$ ) and a mean of .75 (range $=0-2$ ) during probes. Child 2 produced a mean of $1.0 \mathrm{vocal} / \mathrm{verbal}$ comments (range $=0-2$ ) and a mean of 1.0 non-verbal initiations/responses (range $=0-1$ ). Child 3 did not display generalization of either vocal/verbal comments (mean 11.3; range 021) or nonverbal initiations/responses (mean 1.3; range $=0-3$ ). All three participants demonstrated no occurrences of imitative utterances and vocal/verbal questioning during these probes.

\subsection{Generalization 2}

This probe was an unstructured play session with a peer using materials that had been used in earlier intervention sessions. Results revealed that all three participants maintained higher levels of vocal/verbal turn-taking when compared to their baseline data. Only Child 2 maintained higher occurrences of vocal/verbal questioning during baseline and intervention in Generalization 2 probes.

\subsection{Estimate of Effect Sizes}

Percentages of all nonoverlapping data (PAND) [13] was used to evaluate the consistency of experimental effects across participants and to provide an estimate of effect sizes. The resulting calculations for PAND for the high occurrence variables of vocal/verbal turn-taking, initiated and responded vocal/verbal comments, and nonverbal initiations/responses are presented in Table 1 . Additionally, PAND calculations were completed across high occurrence dependent variables to provide an estimate of effect sizes for each participant. The PAND calculations indicated that the parallel talk intervention produced moderate effects in all participants in vocal/verbal turn-taking. 
Table 2. Percentage of all nonoverlapping data (PAND) for each dependent variable across the three participants

\begin{tabular}{lc}
\hline Dependent Variable & PAND \\
\hline & \\
Vocal/Verbal Turn-Taking & $81.01 \%$ \\
I/R Vocal/Verbal Comments & $49.37 \%$ \\
I/R Nonverbal Comments & $49.37 \%$ \\
\hline
\end{tabular}

Note: Generalization 1 and 2 data are not included in the calculations

\subsection{Teacher/ Paraeducator Social Validity}

At the conclusion of the study, the teacher and paraeducators rated their satisfaction with the training by completing a 16 question survey which used a 5point scale (5-strongly agree; 4-agree; 3 do not agree or disagree; 2-disagree; 1-strongly disagree). The mean rating for overall satisfaction was 3.9. These professionals also answered 6 open-ended questions. The three respondents indicated that they believed that the use of parallel talk was worthwhile ("strongly agree"), that the project was worth the additional time required ("agree"), and that they would use parallel talk again ("strongly agree"). One paraeducator commented that children 2 and 3 improved their eye contact during conversations and were more active communicators as a result of the intervention.

\section{Discussion}

This pilot study found that the use of a 5-minute structured parallel talk intervention that occurred during table play between a child and an adult appeared to increase the frequency of verbal turntaking of the three preschoolers who had hearing loss and were delayed in receptive and expressive language and social skills. An increase in the frequency of initiated and responded verbal comments occurred with two of the three children. No changes in the frequency of questions and imitative utterances of adult comments were observed. Generalization of verbal/vocal turn-taking skills occurred with all the participants immediately following intervention (Generalization 1). Furthermore, although it varied by child, generalization was displayed in vocal/verbal turntaking and total initiated and responded verbal/vocal comments in all the children when they played with a peer in an unstructured play time later in the day (Generalization 2).
Before the study, the children displayed little communicative sharing or social interaction. In fact, spontaneous verbal exchanges were often limited to seeking assistance or requesting an adult resolve conflicts with a peer over turns or materials. However, with the parallel talk intervention, the children increased their communicative interactions and social awareness skills as expressed by increased verbal turn-taking and verbal commenting, suggesting that joint attention was enhanced by the adult's use of this strategy. One strength of parallel talk appears to be that because it is a child-directed procedure it permits a child to set the topic, and decide when and how to respond. Because adult comments were always within each child's syntactical level, comprehension was facilitated which may have aided the children in developing the confidence to engage more eagerly in conversations. Since the adult commented regularly about what a child was doing, thinking or feeling, thereby fostering joint attention, a child was able to significantly control the nature of the adult's comments, all of which seemed to make communication exchanges more predictable for a child. This verbal and nonverbal reciprocity is more likely to increase vocabulary for young children as it provides direct labeling of the materials at hand [11]. Because the participants had hearing loss, these types of exchanges may have been missed in infancy and toddlerhood due to the children's inability to utilize auditory cues then.

Since the strategy of parallel talk does not demand communication from a child by providing verbal prompts, verbal models, and asking questions, it reduces emotional pressure to verbally initiate and respond which may have had the effect of increasing verbal turn-taking in the participants. The wait time of 10 seconds between adult comments used in the protocol appeared necessary for allowing a child to hear and process what the adult said about the child's play and for the child to decide if he/she wanted to say. Child 2, the lowest communicator in the study, seemed particularly supported by the approach. Interestingly, in the social validity survey following the study, the paraeducators who implemented parallel talk wrote that they initially found the strategy difficult to learn since questions were not permitted by the protocol and they believed that by asking questions they "helped the children learn." One paraeducator stated that she found parallel talk "unnatural" because she had to continually stop herself from asking questions. It appears that some educators, and speech-language pathologists, automatically assume that by asking questions and providing verbal models, they increase verbal engagement in young children. The results of this 
study may suggest, however, that the opposite may be true. It seems that when educators reduce communicative pressure by increasing wait time, matching linguistic levels, and following the child's verbal and play lead that communicative exchanges increased. Previous literature suggests that parallel talk can be effective when used as one component of a comprehensive communication development program with preschoolers with communicative delays [9]. The current pilot study suggests that parallel talk, used in isolation, appears to offer promise as a facilitative support of language and pragmatic skills in young children with hearing loss, thereby extending the literature. However, since this was a pilot study, clearly additional research is necessary to explore if the outcomes were only a product of the parallel talk intervention, or the unique combination of the program and speech services offered in the oral program.

Based on the observations of this study, the amount of time that parallel talk is used in a classroom may be related to it effectiveness. Although the classroom teacher and paraeducators were told that they could use parallel talk throughout the day after intervention was begun, informal observations revealed that they rarely used it outside of the 5 minute intervention sessions. Yet, the results suggest that the strategy was effective in engaging children in becoming conversational partners. This change was reflected in increases in verbal turntaking, as well as in more verbal comments and more visual and physical connections between the children and their adult communication partner. This was particularly true for Child 2 who dramatically increased her communicative intent as the study progressed. She could be described as a reluctant speaker at the onset of the study, yet with the continued use of parallel talk, her initiated and responded nonverbal communication behaviors increased substantially from baseline levels. Perhaps these effects, and those of the other children, could have been enhanced even more by increasing the length of time that parallel talk was employed.

Even though it was hypothesized that imitative vocal/verbal responses would increase, it is noteworthy that there was little or no spontaneous imitation of adult's comments during the parallel talk intervention phase. Despite this, the teacher and paraeducators reported that all children, at various times, used words or phrases they had heard during the parallel talk intervention sessions at other times of the day. In fact, the classroom teacher commented that Child 1 appeared to "code" the words during intervention and not only use them later in the day but, also on occasion, used them days later. It is possible that the low rate of imitation observed may have been because the adults did not directly request imitation, so the children did not produce it. Yet, when imitation did occur, it appeared from the child's face that the adult had provided a child with an unfamiliar word, and the child was appreciative.

Since improving pragmatics was a critical component of this study the operational definitions required a child to make visual or physical contact in order for a behavior to be coded. In actuality, it was common for the children to engage in conversation while they played, without making visual or physical connections. Because of the way in which definitions were written, it is possible that the present results underestimate the frequency of vocal/verbal turntaking and vocal/verbal comments.

\subsection{Generalization of Communicative and Social Skills}

Adequate generalization of specific pragmatic skills was displayed by the three participants in both of the generalization probes. All participants displayed higher than baseline occurrences of verbal/vocal turn-taking during Generalization 1 and Generalization 2 probes. Additionally, 2 of the 3 participants displayed higher than baseline occurrences of verbal/vocal commenting in both Generalization 1 and 2 probes. Surprisingly, Child 3, who did not show an increase in verbal comments during intervention, displayed the highest levels of verbal turn-taking and verbal comments during Generalization 1 probes (immediately following training). This unanticipated result appears to be related to this child's desire to re-engage the paraeducator after she stopped using the parallel talk intervention. This child occasionally expressed dissatisfaction through words, facial expressions and body language when he was unsuccessful in getting the paraeducator to narrate his play during this probe. Because of his communication level, this child may have had difficulty generating the appropriate vocabulary without the indirect support which parallel talk provided, making his struggle to invite the paraeducator back into his play more compelling.

\subsection{Limitations}

There are limitations in the present study that should be acknowledged. First, the results may have been more consistent and powerful if the period of intervention could have been lengthened and the use of parallel talk had been distributed throughout the day. As others have commented, intensity of instruction matters [8]. Moreover, the effects may have been more robust if this procedure had been 
carried out for a full school year. Second, although efforts were made to select children with similar communicative levels, the outcomes are limited to the unique characteristics of the children who participated. Because participants received daily oral language instruction and speech therapy, it may be that the outcomes were influenced by these services and not merely by the parallel talk intervention. Finally, it should be noted that the choice of materials may have influenced the quality and rate of communication behaviors noted in this study. It appeared that some materials were more amenable to verbal interactions than others. Future research should compare the efficacy of using parallel talk with prelinguistic groups of young children to see if it fosters joint attention with them. Also, it would be useful to determine if children with other disabilities, and with intellectual involvement, respond to the use of parallel talk. Examining the utility of using parallel talk, paired with direct instruction, such as the use of mands, might extend the practical application of this strategy and is also worthy of study.

\subsection{Implications for Practice}

Professionals who work with young children are committed to employing evidence-based practices in serving young children [15]. Identifying researchbased strategies allows educators to aggressively intervene in areas that children with hearing loss find most problematic. The outcomes of this pilot study suggest that the use of parallel talk may hold promise as a way of addressing some of the communication and social needs of these children. Parallel talk is an indirect, naturalistic facilitation strategy that does not demand that a child imitate, respond to questions or commands, or perform verbally upon request. Since parallel talk allows a child to "take charge of a verbal interaction," it encourages a child to guide the conversation in the direction in which the child feels most comfortable, is familiar with the vocabulary, or is simply interested. Although the strategy does not require a child to speak or use appropriate pragmatics, it appears to indirectly model verbal turntaking and gives a child words for what matters to the child most at any given moment - what the child is doing, thinking, or feeling at that moment.

\subsection{Acknowledgements}

We express gratitude to Janet Knust, Lindsey Selfridge, and Marcy Weiss for their assistance with this study.

\section{References}

[1] Z. Abu Bakar, P. Brown, and M. Remine, "Sensitivity in interactions between hearing mothers and their toddlers with hearing loss: The effect of cochlear implantation." Deafness \& Education International, 12(1), 2010, pp. 2-15.

[2] A. Boothroyd, A. Geers, and J. Moog, J. S. "Practical Implications of Cochlear Implants in Children." Ear \& Hearing, 12(4)(SUPPLEMENT), 1991, pp. 81S-89S.

[3] S. Camarata, P. Yoder, P., and M. Camarata, M. (2006). "Simultaneous treatment of grammatical and speech-comprehensibility deficits in children with Down syndrome. “ Down Syndrome: Research \& Practice, 11(1), 2006, pp. 9-17.

[4] E. Delaney, S. Ezell, N. Solomon, T. Hancock, T., and A. Kaiser. The KIDTALK Behavior and Language Code: Manual and coding protocol. George Peabody College for Teachers, Nashville, TN., USA, 1997.

[5] A. Geers, J. Moog, J. Biedenstein, C. Brenner,and H. Hayes. "Spoken language scores of children using cochlear implants compared to hearing age-mates at school entry." Journal of Deaf Studies and Deaf Education, 14(3),2009, pp. 371-384.

[6] A. Honig. "Talk, read, joke, make friends: Language power for children.” DayCare and Early Education, 1998, Spring,1989, pp. 14-17.

[7] A. Honig. "Language flowering, language empowering: 20 ways parents and teachers can assist young children. “ Montessori Life, 2001, Fall, pp. 31-35.

[8] A. Kaiser, T. Hancock, and J. Nietfeld, J. P. "The effects of parent-implemented enhanced Milieu Teaching on the social communication of children who have autism." Early Education and Development, 11(4), 2000, pp. 23-446.

[9] A. Kaiser, T. Hancock, and J. Trent. "Teaching parents communication strategies. "Early Childhood Services: An Interdisciplinary Journal of Effectiveness, 1(2), 2007, pp. 107-136.

[10] S. Landry, and K. Loveland. "Communication behaviors in autism and developmental language delay." Journal of Child Psychology and Psychiatry, 29(5), 1998, pp. 621-634.

[11] A. McDuffie, P. Yoder, and W. Stone, W. L. (2006). "Labels increase attention to novel objects in children with autism and comprehension-matched children with typical development. “Autism, 10(3), 2006, pp. 288-301.

[12] P. Mundy, and A. Gomes. "Individual differences in joint attention skill development in the second year." Infant Behavior and Development, 21(3), 1998, pp. 469-482. 
[13] R. Parker, S. Hagan-Burke, and K. Vannest. "Percentages of all non-overlapping data (PAND): An alternative to PND. Journal of Special Education, 40, 2007, pp. 194-204.

[14] C. Peterson. "Theory-of mind development in oral deaf children with cochlear implants or conventional hearing aids." Journal of Child Psychology and Psychiatry, 45(6), 2004, pp. 1096-1106.

[15] S. Sandall, M. Memmeter, B. Smith,and M. McLean, M. DEC recommended practices: A comprehensive guide for practical application in early intervention/Early childhood special education. Longmont, CO: Sopris West, 2005.

[16] S. Tasker, M. Nowakowski,and L. Schmidt. "Joint attention and social competence in deaf children with cochlear implants. "Journal of Developmental and Physical Disabilities, 22(5), 2010, pp. 509-532.

[17] S. Ulvund, and L. Smith, L. (1996). "The predictive validity of nonverbal communicative skills in infants with perinatal hazards." Infant Behavior and Development, 19(4), 1996, pp. 441-449. 